\title{
Jährliche Meldung für den Umgang mit Transplantaten nach Artikel 18 Absatz 1 des Bundesbeschlusses über die Kontrolle von Transplantaten neu via Internet möglich
}

Bundesamt für Gesundheit

Korrespondenz:

Barbara Schärer, lic. phil. nat. Bundesamt für Gesundheit Facheinheit Biomedizin

Sektion Transplantation + Forschung am Menschen

CH-3003 Bern

Tel. 0313226322

E-Mail:

barbara.schaerer@bag.admin.ch
Seit 1996 werden Transplantate im Bundesbeschluss vom 22. März 1996 über die Kontrolle von Transplantaten (SR 818.111) und der entsprechenden Verordnung vom 26. Juni 1996 über die Kontrolle von Transplantaten (SR 818.111.3) geregelt.

Als Transplantate werden die zur Übertragung auf den Menschen bestimmten Organe, Zellen oder Gewebe menschlichen oder tierischen Ursprungs definiert. Dazu gehören insbesondere auch Knochen, Cornea, Blutstammzellen (inklusive Nabelschnurblut zum Zweck der Gewinnung und anschliessender Lagerung von Stammzellen) sowie generell alle ganzen, vitalen Zellen (z. B. echte Frischzellentherapie). Der Umgang umfasst insbesondere Entnahme, Lagerung, Ein- und Ausfuhr, Inverkehrbringung und Transplantation (Art. 3 Bst. b).

Für den Umgang mit menschlichen Transplantaten gilt gemäss Artikel 18 des Bundesbeschlusses eine Melde- und eine Bewilligungspflicht. Wer menschliche Transplantate entnimmt, lagert, in Verkehr bringt oder transplantiert muss dem Bundesamt für Gesundheit (BAG) Meldung erstatten (Art. 18 Abs. 1). Diese Meldung muss nach Artikel 22 der Verordnung vor Aufnahme der Tätigkeit erstattet werden und muss jährlich wiederholt werden. Nach Artikel 23 der Verordnung muss die jährliche Meldung die Art und Anzahl der entnommenen, transplantierten und in Verkehr gebrachten Transplantate sowie die verwendeten Tests beinhalten. Bei der Lagerung von Stammzellen müssen zudem jährlich die Anzahl aller Ein- und Ausgänge sowie die Anzahl der gelagerten Dosen gemeldet werden. Diese jährlichen Meldungen werden jeweils bis zum 31. März des Folgejahres für ein abgelaufenes Kalenderjahr erwartet. Die Unterlassung der Meldepflicht gemäss Artikel 18 des Bundesbeschlusses unterliegt den Strafbestimmungen (Art. 33) und kann mit Haft oder Busse bestraft werden.
Der Bundesbeschluss und die Verordnung über die Kontrolle von Transplantaten können beim Bundesamt für Bauten und Logistik, Abteilung EDMZ (Vertrieb), Sektion Verkauf, 3003 Bern (Telefon 03132550 50, Fax 03199200 23/24), bezogen oder im Internet konsultiert werden (www.bag.admin.ch/transpla/d/, Stichwort: Gesetzestexte).

\section{Meldung via Internet}

Um die jährlichen Meldungen über den Umgang mit Transplantaten gemäss Artikel 18 Absatz 1 des Bundesbeschlusses zu erleichtern, können diese neu gesichert per Internet auf der Seite www.txdb.admin.ch getätigt werden. Zudem können die Tätigkeiten bereits Anfang Jahr für das laufende Jahr vorerfasst und kontinuierlich via Internet aktualisiert werden und müssen Anfang des nächsten Jahres nur noch abgeschlossen, das Formular ausgedruckt und dem BAG unterschrieben zugeschickt werden.

Auf der Startseite der Meldung via Internet können im Impressum die benötigten Anforderungen an das Computersystem und die Grösse der Verschlüsselung nachgeschaut werden. Zudem ist auf der Startseite eine Anleitung für die Meldung via Internet abrufbar (durch Anklicken des «?»). Diese kann auch beim BAG direkt verlangt werden.

Aus programmiertechnischen Gründen erscheint der Startbildschirm der Datenbank zuerst auf Deutsch, aber die Sprache kann durch Anklicken von «Französisch» auf Französisch geändert werden. Nach dem Anmelden kann zudem die Sprache des Benutzers (Deutsch oder Französisch) eingestellt werden und die Texte erscheinen in der gewählten Sprache. Nach der Änderung im Benutzerprofil muss bei jedem späteren Einloggen die Sprache nicht jedes Mal neu eingestellt werden. 
Die im Internet getätigte Meldung kann direkt auf einem Formular ausgedruckt werden. Dieses ausgedruckte Formular muss unterschrieben an das BAG an die aufgedruckte Adresse geschickt werden. Aus technischen Gründen ist eine Authentifizierung in dieser Art noch notwendig.

Auf Anfrage (schriftlich oder per Email an: Bundesamt für Gesundheit, Facheinheit Biome- dizin, Sektion Transplantation und Forschung am Menschen, 3003 Bern, E-Mail: txdb@bag. admin.ch) werden den Meldenden ein Benutzername und ein Passwort zugeteilt, mit denen sie sich einloggen können.

Für Fragen bezüglich der Meldung Ihrer Transplantationstätigkeit steht Ihnen Frau B. Schärer unter der Korrespondenzadresse gerne zur Verfügung.

\section{Anleitung zum Melden der Transplantations- tätigkeit via Internet}

\section{Einleitung: Sprache der Datenbank}

Aus programmiertechnischen Gründen erscheint der Startbildschirm der Datenbank auf Deutsch, aber Sie können die Sprache durch Anklicken von «Französisch» auf Französisch ändern. Nachdem Sie sich in der Datenbank angemeldet haben, können Sie ebenfalls unter «Benutzerprofil» die Sprache der Datenbank auf «Französisch» wechseln und sämtliche Texte erscheinen nun auf Französisch.

Standardmässig ist bei jedem Benutzer die Sprache zu Beginn auf Deutsch eingestellt. Nachdem Sie jedoch die Benutzersprache auf Französisch geändert haben, erscheint bei jedem späteren Einloggen die Datenbank auf Französisch und Sie müssen die Sprache nicht jedes Mal neu einstellen.

\section{Anmeldung \\ (Benutzername und Passwort)}

Auf Anfrage (schriftlich oder per Email an: Bundesamt für Gesundheit, Facheinheit Biomedizin, Sektion Transplantation und Forschung am Menschen, 3003 Bern, E-Mail: txdb@bag. admin.ch) werden Ihnen ein Benutzername und ein Passwort zugeteilt.

- Um sich anzumelden, klicken Sie auf das entsprechende Eingabefeld: «Anmelden».

Korrespondenz:

Bundesamt für Gesundheit Facheinheit Biomedizin Sektion Transplantation und Forschung am Menschen CH-3003 Bern

E-Mail: txdb@bag.admin.ch
- Auf der nun erscheinenden Maske geben Sie Ihren Benutzernamen und das Passwort in die entsprechenden Felder ein. Gross- und Kleinschrift werden bei beiden nicht berücksichtigt.

- Klicken Sie nun auf das Feld «Anmelden».
- Es erscheint die Meldung: «Sie wurden erfolgreich angemeldet».

- Klicken Sie nun auf das Feld «Verlassen».

Bei Ihrer ersten Anmeldung werden Sie vom System zu einer Änderung des Passwortes aufgefordert. Das neue Passwort darf nicht gleich dem alten sein und es muss länger als 5 Zeichen sein. Bei Bedarf können Sie das Passwort auch später jederzeit ändern. Bei Verlust Ihres Passwortes wird Ihnen das BAG auf Antrag ein neues erteilen.

Bei dreimaliger falscher Eingabe des Passwortes werden Sie vom System automatisch gesperrt. Zur Entsperrung müssen Sie sich beim BAG melden.

\section{So melden Sie Ihre Tätigkeiten mit Transplantaten gemäss Art. 18 Abs. 1 des Bundesbeschluss sowie Art. 23 Abs. 2 und 3 der Verordnung über die Kontrolle von Transplantaten via Internet}

- Klicken Sie auf das Eingabefeld «Meldungen verwalten».

- Auf der nun erscheinenden Maske klicken Sie auf «Einfügen».

- Auf der nun erscheinenden Maske («Meldungen bearbeiten») geben Sie als erstes das Jahr ein, für das Sie melden wollen (= vergangenes Kalenderjahr oder Vorerfassung für das laufende Jahr). Die Abteilung und Ihr Meldevisum sind bereits automatisch ausgefüllt. Das Meldedatum wird ebenfalls automatisch ausgefüllt, sobald Sie «Speichern» drücken. Den «Meldestatus» können Sie auf 
«Vorerfasst» belassen, falls Sie die Meldung noch nicht definitiv abschliessen und später noch weitere Daten hinzufügen möchten. Falls dies nicht der Fall sein sollte, können Sie bereits jetzt den «Meldestatus» auf «Erfasst» ändern. Zusätzlich können Sie im Bemerkungsfeld allfällige Bemerkungen hinschreiben.

- Klicken Sie jetzt auf «Speichern».

- Es erscheint die Meldung «Datensatz erfolgreich gespeichert» und im unteren Teil der Maske haben Sie nun die Möglichkeit, die zu meldenden Handlungen und Tests einzufügen. Klicken Sie auf «Einfügen» (in Ausnahmefällen, bei Meldepflichtigen, die nicht Handlungen und Tests melden müssen, erscheint nach dem Speichern ein Textfeld, in das die Meldung eingegeben werden kann).

- In der erscheinenden Maske bei den «Handlungen» können Sie jetzt die «Handlungsart» (Entnahme, Transplantation, Lagerung usw.) und die «Transplantatart» (Cornea, Blutstammzellen, Leber usw.) aus den vorgegebenen Listen auswählen und die entsprechende Anzahl der Handlungen eingeben. Im Bemerkungsfeld können Sie allfällige Bemerkungen wie die Herkunft der Transplantate oder Ergänzungen zu der Transplantatart eingeben.

- Klicken Sie jetzt auf «Speichern» (Sie können nach dem Speichern immer noch korrigieren und erneut speichern).

- Es erscheint die Meldung «Datensatz erfolgreich gespeichert». Falls dieser Datensatz in Ordnung ist, klicken Sie nun auf «Verlassen», um einen neuen Datensatz einzugeben. Sie gelangen wieder in die Maske der Meldung. Für die Eingabe weiterer Handlungen gehen Sie gleich vor.

- Beim Einfügen von Tests erscheint eine Maske, in der Sie die «Testart» aus der vorgegebenen Liste auswählen können. Die «Testnummer» wird automatisch generiert, jedoch müssen Sie den verwendeten Test («TestKit») selber eingeben. Klicken Sie nun auf «Speichern».

- Es erscheint die Meldung «Datensatz erfolgreich gespeichert». Falls dieser Datensatz in Ordnung ist, klicken Sie nun auf «Verlassen», um einen neuen Datensatz einzugeben. Sie gelangen wieder in die Maske «Meldungen bearbeiten». Für die Eingabe der weiteren durchgeführten Tests gehen Sie gleich vor.

- In der Maske «Meldungen bearbeiten» erscheinen nun alle Ihre eingegebenen Handlungen und Tests. Falls Sie diese noch ändern möchten, können Sie auf das Symbol «Lupe» neben dem zu ändernden Eintrag klicken und es erscheint die entsprechende Eingabemaske mit Ihren Angaben, die Sie nun ändern können.

- Wenn alle Angaben korrekt sind, können Sie die Meldung durch Anklicken von «Speichern» bestätigen.

- Es erscheint die Meldung «Datensatz erfolgreich gespeichert».

- Wenn die Meldung abgeschlossen werden soll, ändern Sie bitte den Meldestatus auf «Erfasst».

- Durch Anklicken von «Drucken» kann die Meldung auf einem Formular ausgedruckt werden.

- Bitte schicken Sie das ausgedruckte und unterschriebene Formular an das BAG an die angegebene Adresse. Aus technischen Gründen ist eine Authentifizierung in dieser Art noch notwendig.

- Nachdem die Meldung mit «Erfasst» abgeschlossen und das Formular an das BAG geschickt wurde, wird es von diesem geprüft und anschliessend der Meldestatus in der Datenbank auf «Geprüft» gesetzt.

\section{Kontinuierliches Erfassen von Tätigkeiten}

Zur Vereinfachung der jährlichen Meldung können Sie im Status «Vorerfasst» beliebig oft die Meldungen abändern und ergänzen. Sie können also Ihre Tätigkeiten bereits Anfang Jahr für das laufende Jahr vorerfassen, kontinuierlich im Internet aktualisieren und müssen dann Anfang des nächsten Jahres nur noch die Meldung abschliessen, indem Sie den Status auf «Erfasst» ändern, das Formular ausdrucken und dem BAG unterschrieben zuschicken.

\section{Ändern einer Meldung}

Erfasste Meldungen können geändert werden, solange diese vom BAG noch nicht geprüft wurden. Eine geänderte Meldung muss erneut an das BAG geschickt werden.

- Anklicken des Feldes «Meldungen verwalten».

- Anklicken von «Suchen».

- Es erscheint nun die Maske mit der Übersicht aller für Ihre Abteilung je getätigten Meldungen. Durch Anklicken des Symbols «Lupe» neben einer Meldung gelangen Sie in die Eingabemaske dieser Meldung. Solange der Status «Erfasst» ist, können Sie die Meldung wie zuvor beschrieben abändern, neu speichern und ans BAG schicken. 
- Falls der Status bereits «Geprüft» ist, können Sie die Meldung nur noch anschauen und ausdrucken. Sie müssen nun jede Änderung dem BAG schriftlich oder per E-Mail melden. Die Meldung wird danach vom BAG revidiert.

\section{Stammdaten}

Sie können Ihre Stammdaten (Angaben zum Institut, der Abteilung und der verantwortlichen Person) jederzeit einsehen durch Anklicken von «Stammdaten», jedoch nicht selber abändern. Falls Angaben nicht korrekt sind oder Sie Ergänzungen anbringen möchten, bitten wir Sie, die Änderungen oder Ergänzungen dem BAG zu melden (per E-Mail oder schriftlich).

\section{Angaben im Benutzerprofil}

Sie können die Angaben Ihres Benutzerprofils jederzeit aufrufen oder, mit Ausnahme des Benutzernamens, Status, Benutzergruppe und Abteilung abändern.

- Klicken Sie dazu auf «Benutzerprofil».

- Sie können die Sprache auf «Deutsch» oder «Französisch» einstellen.

- Bei der «Seitengrösse» können Sie nach Ihrem Bedürfnis einstellen, wieviele Suchergebnisse auf einer Seite dargestellt werden sollen. Bei der empfohlenen Bildschirmauflösung $(1024 \times 768)$ ist «20» ein Standardwert.

- Als weiteres können Sie Ihren Namen, die Telefonnummer, Mobilenummer und $\mathrm{Ihr}$ E-Mail eintragen oder abändern.

- Die Änderungen können Sie mit «Speichern» übernehmen.

\section{Weitere Fragen}

Die Mitarbeitenden des BAG, Facheinheit Biomedizin, Sektion Transplantation und Forschung am Menschen, erteilen Ihnen gerne weitere Auskünfte. 